\title{
Stage 1B2 Invasive Cervical Cancer diagnosed at 20 Weeks Gestation: Neoadjuvant Chemotherapy followed by Caesarean Radical Hysterectomy at Term and Chemoradiation: Report of a Case
}

\author{
*Thomas O. Konney ${ }^{1}$, Carolyn Johnston ${ }^{2}$, Kwabena Amo-Antwi', Adu Appiah-Kubi', \\ Augustine Tawiah'1, Akwasi Antwi-Kusi' ${ }^{2}$, Ernest O. Bonsu ${ }^{3}$, Alexander T. Odoi ${ }^{1}$ \\ ${ }^{1}$ Department of Obstetrics and Gynaecology, Gynaecologic Oncology Unit, Komfo Anokye \\ Teaching Hospital, Kumasi, Ghana \\ ${ }^{2}$ Department of Anaesthesia and Intensive Care, Komfo Anokye Teaching Hospital, Kumasi, \\ Ghana \\ ${ }^{3}$ Radiation Oncology Unit, Komfo Anokye Teaching Hospital, Kumasi Ghana \\ ${ }^{4}$ Division of Gynecologic Oncology, University of Michigan, USA
}

\section{*Corresponding Author: Thomas O. Konney, E-mail address: tom.konney@yahoo.com}

\section{Background}

The diagnosis and treatment of cervical cancer during pregnancy is a challenge that requires a multi-disciplinary approach. The management depends on the gestational age at diagnosis, the stage of disease, the woman's desire to continue the pregnancy and desire to preserve fertility. Neoadjuvant chemotherapy (NACT) is a treatment option for women diagnosed before 24 weeks gestation who wish to continue the pregnancy. Our objective was to present the first such case in West Africa.

\section{Methods}

We present a 40-year old G3P2 with stage IB2 poorly differentiated squamous cell carcinoma of the cervix diagnosed at 20-weeks' gestation. She received 3 cycles of NACT with Cisplatin $75 \mathrm{mg} / \mathrm{m} 2$ and Paclitaxel $135 \mathrm{mg} / \mathrm{m} 2$ followed by radical caesarean hysterectomy and pelvic lymphadenectomy at 37 weeks and 4 days. We reviewed the relevant literature for similar cases.

\section{Outcomes}

The tumour size shrank by $50 \%$ by the third cycle and bleeding ceased by the start of the second cycle. The baby weighed $2.2 \mathrm{~kg}$ with good Apgar scores. Histopathology showed resection margins to be free of disease, positive for LVSI, tumour size of $4 \mathrm{~cm}$ and residual tumour in bilateral pelvic lymph nodes. The patient completed whole pelvic radiation and brachytherapy. Both mother and infant are doing well at 10 months posttreatment and are in surveillance.

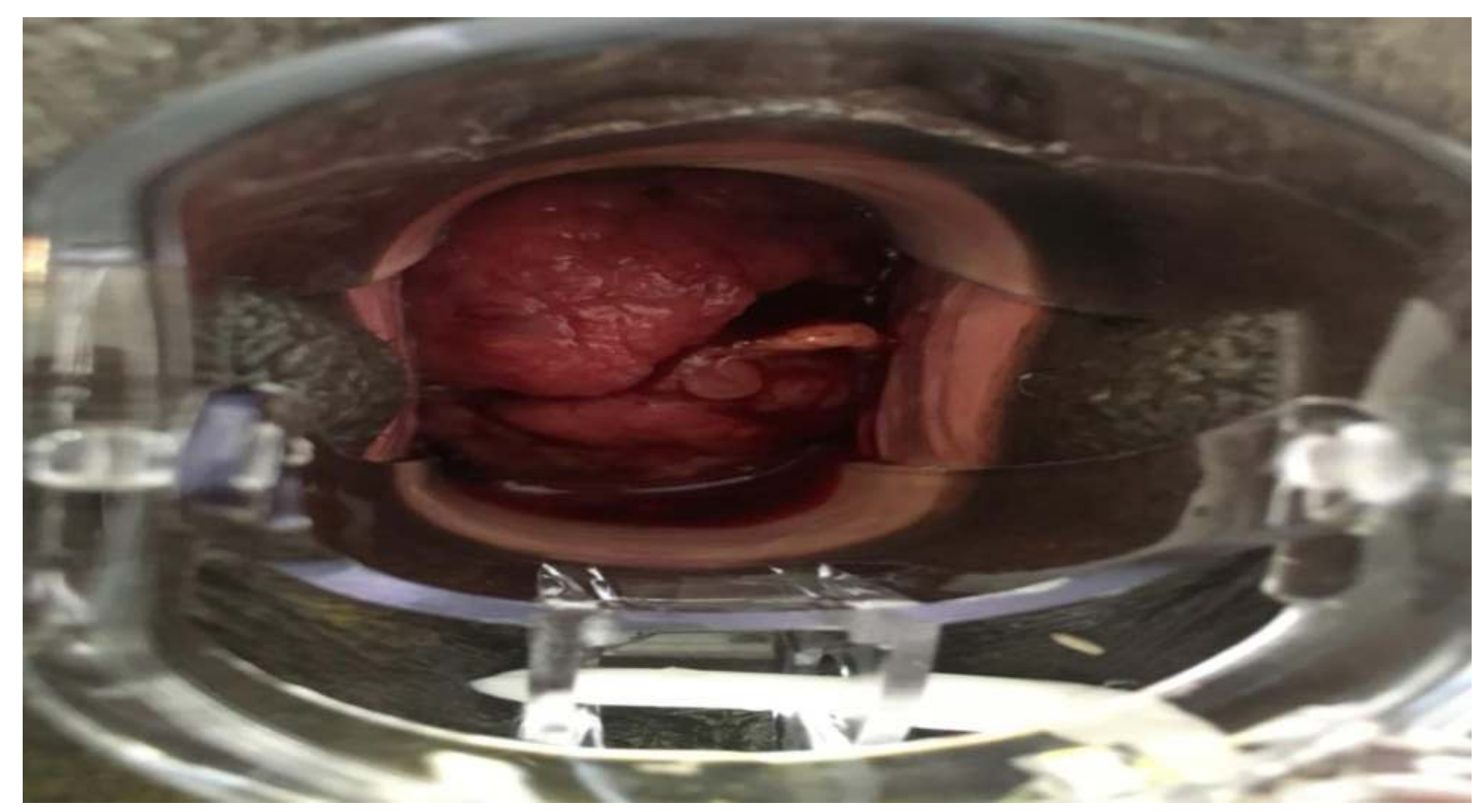

Figure 1: Speculum examination showing cervical cancer

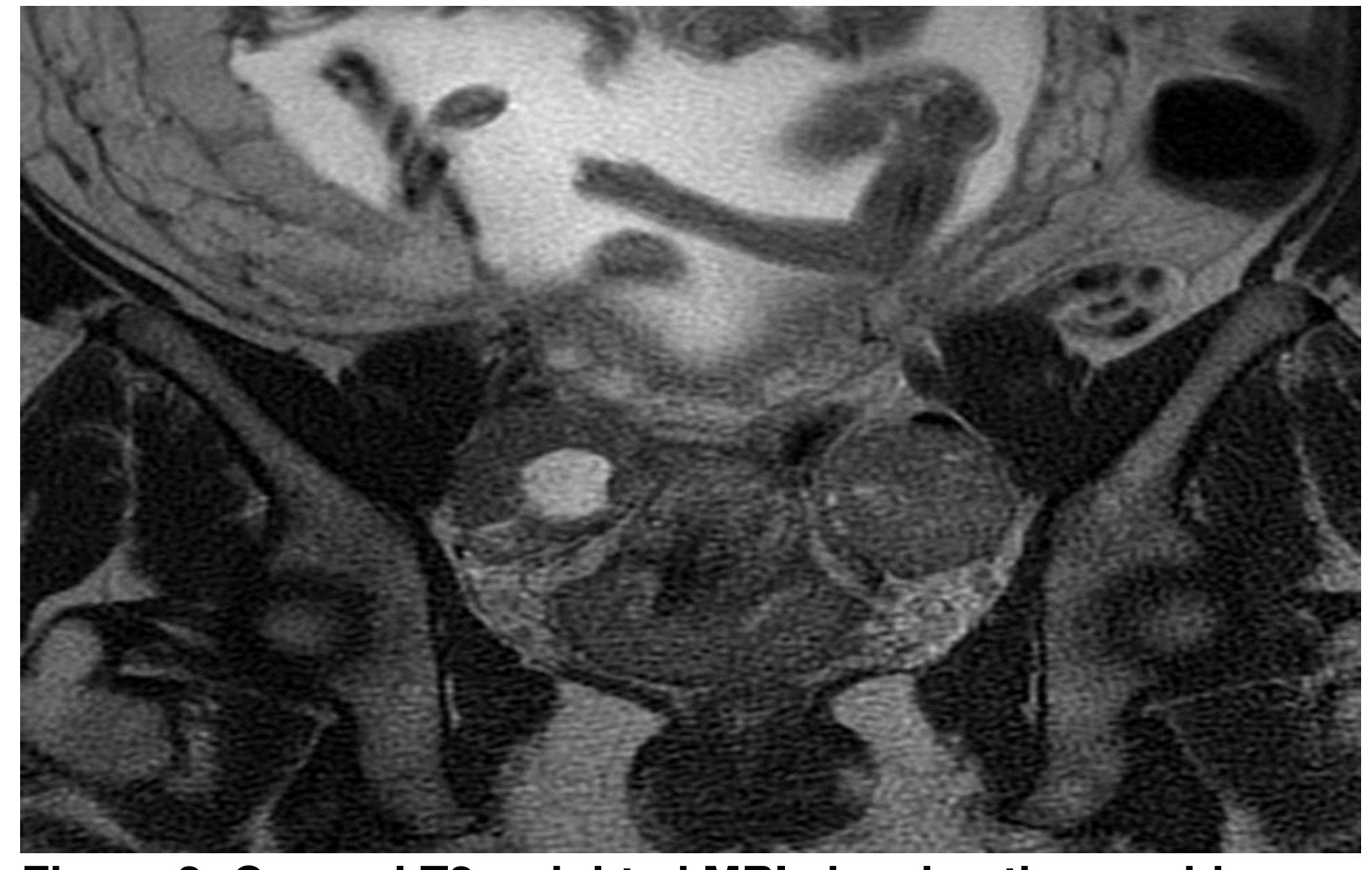

Figure 2: Coronal T2 weighted MRI showing the gravid Uterus with a bulky cervix containing an ill-defined mass

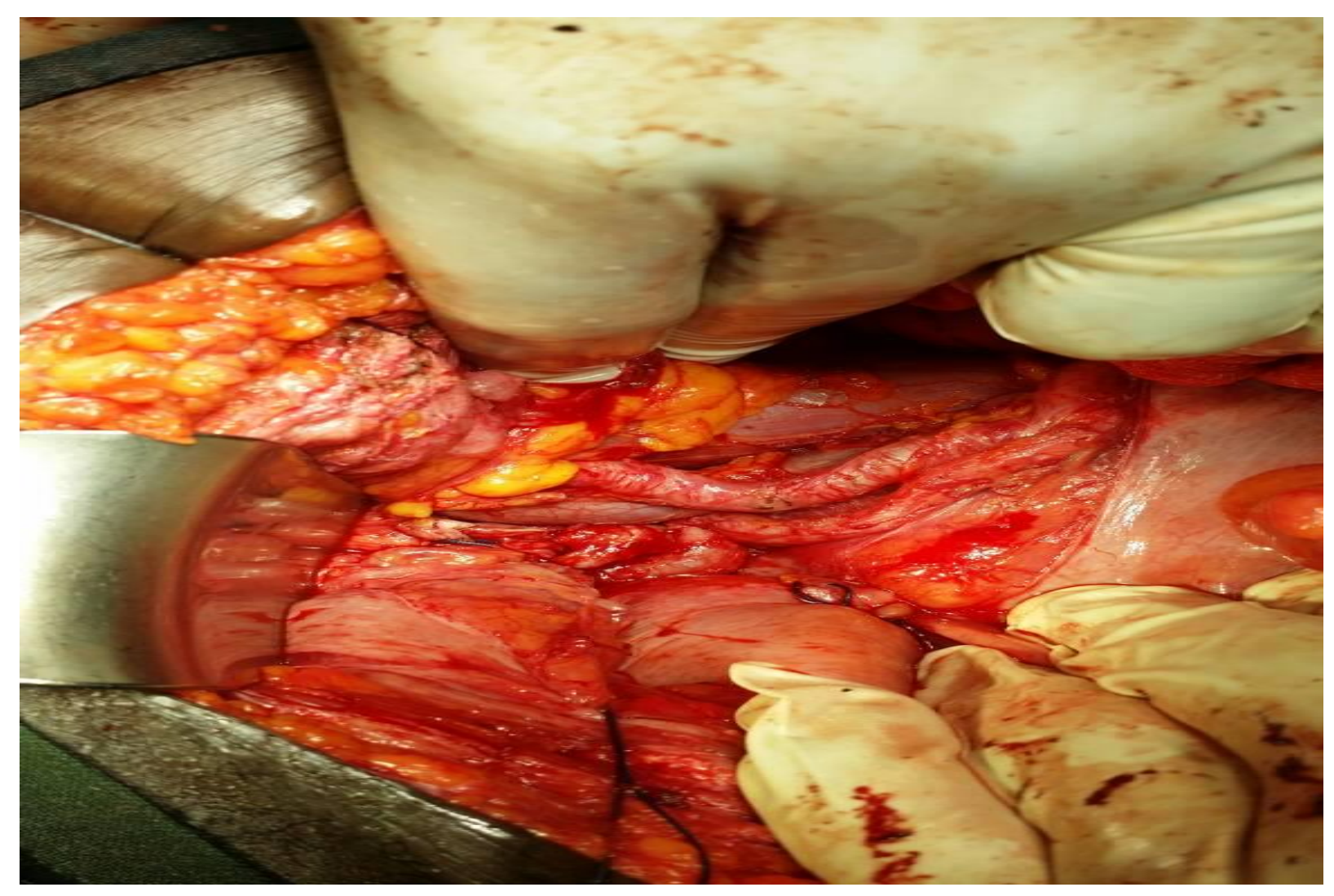

Figure 3: Pelvic lymph node dissection

\section{Conclusions}

We demonstrate that NACT followed by caesarean radical hysterectomy is a safe option to delay delivery of the baby in the management of locally invasive cervical cancer first diagnosed in pregnancy. NACT led to cessation of vaginal bleeding and tumour shrinkage. 